\title{
Biologic Risk Code
}

National Cancer Institute

\section{Source}

National Cancer Institute. Biologic Risk Code. NCI Thesaurus. Code C95352.

A coded value specifying the risk associated with the biologic. 